\title{
Adsorption Characteristics of Zeolite A Synthesized from Wassa Kaolin for Thermal Energy Storage
}

\author{
Linus Kweku Labik ${ }^{1}$, Bright Kwakye-Awuah ${ }^{1}$, Eric Kwabena Kyeh Abavare ${ }^{1}$, Baah Sefa-Ntiri ${ }^{2}$, Isaac Nkrumah $^{1}$ \\ \& Craig Williams ${ }^{3}$ \\ ${ }^{1}$ Department of Physics, Kwame Nkrumah University of Science and Technology, Kumasi, Ghana \\ ${ }^{2}$ Department of Physics, University of Cape Coast, Cape Coast, Ghana \\ ${ }^{3}$ Faculty of Science and Engineering, University of Wolverhampton, Wolverhampton, WV1 1LY, United \\ Kingdom
}

Correspondence: Linus Kweku Labik, Department of Physics, Kwame Nkrumah University of Science and Technology, Kumasi, Ghana. Tel: 233-26-232-6483, E-mail: linuslabik@gmail.com

Received: June 5, 2020

doi:10.5539/jmsr.v9n3p21
Accepted: July 2, 2020

Online Published: September 30, 2020

\begin{abstract}
Zeolites based on the numerous applications can be utilised in providing solutions to some challenges of our world. With the ability to store thermal energy as chemical potential, zeolites are able to store thermal energy for long periods. This can occur with very minimal loss of energy and indefinitely unless the zeolite comes into contact with an adsorbate. The use of zeolite - water as adsorbent - adsorbate pair in thermal energy storage (TES) applications have been studied and have shown good results. However, the cost of zeolites synthesized from reagents continue to hamper the effective use of this adsorbent. Zeolite A was synthesized from kaolin from Wassa in Ghana based on a modified synthesis route. The adsorption properties of the zeolite utilising a designed and fabricated TES system using amounts of $100 \mathrm{~g}, 200 \mathrm{~g}, 300 \mathrm{~g}, 400 \mathrm{~g}$ and $500 \mathrm{~g}$ of zeolite with a 1:1.5 ratio to water. Adsorption isosteres were plotted with the temperature and pressure values recorded and results showed correlation to adsorption behaviour of zeolites. Langmuir adsorption isotherms with r-squared values greater than $90 \%$ confirmed the affinity of water for zeolites. isosteric heat of adsorption was calculated with the minimum being $5,655.84 \mathrm{~J} / \mathrm{g}$ and the maximum being 8,113.44 J/g. This confirms that the Zeolite A synthesized from Was kaolin has the structural properties needed for TES applications.
\end{abstract}

Keywords: thermal energy storage, zeolite, adsorption, adsorption isotherm. heat pump

\section{Introduction}

As society develops, the range of energy applications continue to increase with energy consumption. This results in a mismatch when generation doesn't meet demand. This gap can be reduced or eliminated via energy storage. Energy storage is critical to efficient utilization of energy especially thermal energy use (Paksoy, 2005). Thermal energy is favoured due to its minimal effect on the environment though difficult to store. Thermal Energy Storage (TES) due to its flexibility can improve energy supply, reduce consumption and store excess energy(Ding \& Riffat, 2013; Schreiber, Lanzerath, Reinert, Grüntgens, \& Bardow, 2016). TES systems can utilize solar energy, geothermal energy as well as waste heat from domestic and industrial processes (Gwadera \& Kupiec, 2014). TES employing sorption utilizes the reversible reactions involved in the storage of thermal energy. The physical and chemical bonds of the sorbent and sorbate are used to store the energy (Scapino, Zondag, Van Bael, Diriken, \& Rindt, 2017).

In adsorption, no chemical reaction occurs as the molecules of the adsorbate are attached to the adsorbent material. Energy is instead lost as this reaction is exothermic (Dicaire \& Tezel). Addition of heat during the desorption phase results in an endothermic reaction with the separation of adsorbate and adsorbent (Scapino et al., 2017). This process also known as physisorption is reversible.

The need to develop materials better suited for applications such as thermal energy storage has become compelling. Many materials such as silica gels, silicoaluminophosphates (SAPOs) and zeolites have been suggested (Herzog, Jänchen, Kontogeorgopoulos, \& Lutz, 2014). Water has been mostly used as the adsorbate due to its high latent heat and nonpolluting nature (Chan, Chao, Sze-To, \& Hui, 2012). 
A working pair for thermal energy adsorption should meet some basic requirements. The adsorbate should be nontoxic, inflammable, ecofriendly and have high latent heat. Adsorbents are also expected to have large adsorption capacities, good thermal stability and high affinity for the adsorbate ( $\mathrm{Li}, 2013)$. The wide application of zeolites besides silica gel has led to innovative designs of heat exchanger systems as well as increasing optimization of the material (Dawoud, 2013; J. Janchen \& Stach, 2011).

The properties of zeolites such as hydrothermal stability is dependent on some factors. These are the $\mathrm{Si} / \mathrm{Al}$ ratio, nature of cations, lattice types as well as cavities and their sizes (Jochen Janchen et al., 2012). Zeolites are crystalline aluminosilicates and used in various industrial processes. Their three-dimensional unit framework is as a result of the $\mathrm{SiO} 4$ and $\mathrm{AlO} 4$ tetrahedra (Khan, Arafat, Reza, Razzaque, \& Alam, 2010). The reversible adsorption and desorption processes of zeolites has been well documented. This thermal property is critical in the exploitation of zeolites in TES (Vieillard, 2010). This has contributed to the increasing research on zeolites for TES applications.

Several data exist for zeolites synthesized from reagents and some natural zeolites. With the desire to reduce the cost of zeolites, the use of local raw materials in synthesis is imperative. The use of kaolin as starting material has been extensively researched on. However, the use of kaolin from Wassa, Ghana, was recently explored by (VonKiti, 2016). The thermo- physical and thermal properties of zeolites synthesized from this type of kaolin will contribute to the efficient exploitation of this resource as in Figure 1

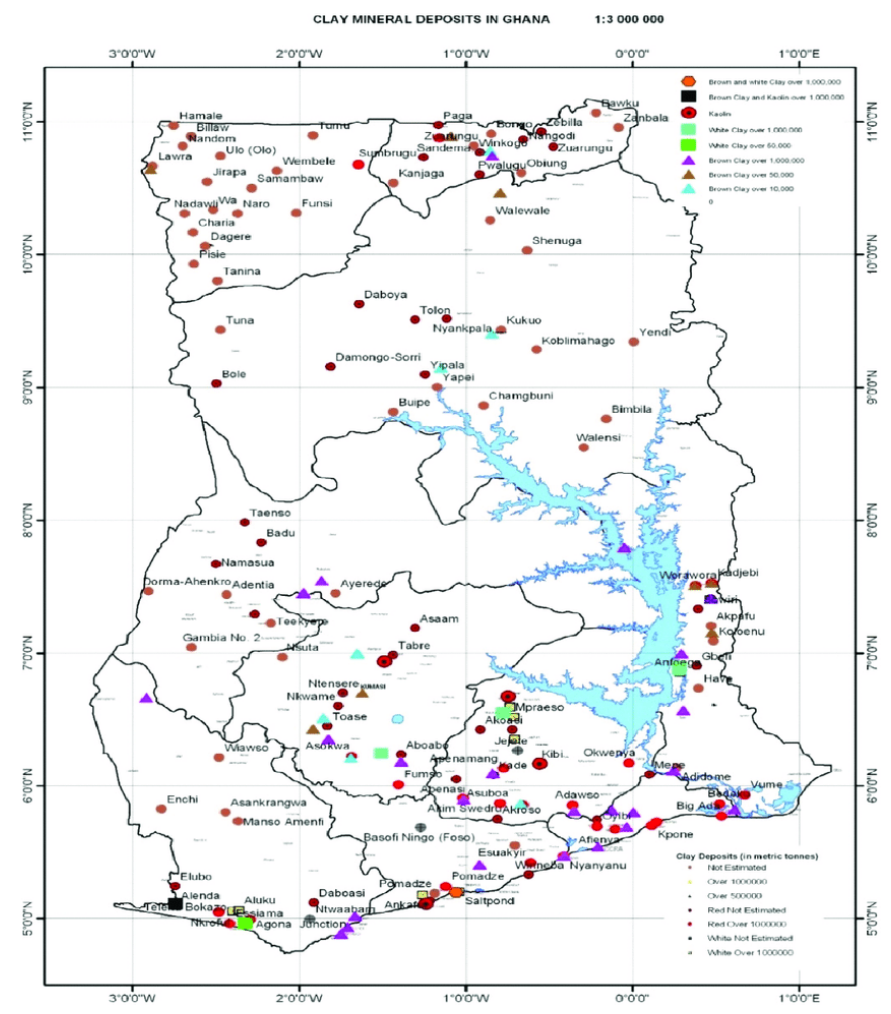

Figure 1. Map of clay deposits in Ghana (Asamoah et al., 2018)

\section{Principle of Operation}

Sorption has been used to describe the process as gas or vapour is fixed on the surface of a liquid or solid. The captured substance is the sorbate and the other sorbent. Sorption includes both adsorption and absorption as they involve mass or heat transfer although different phenomena (Ding \& Riffat, 2013). Sorption storage of energy utilizes the reversible reactions between the sorbate and sorbent and can provide a lossless heat storage (van Alebeek et al., 2018). In adsorption, the adsorbate is amassed onto the surface of the adsorbent depending on the regions which are most attractive as in Figure 2.

Heat is adsorbed during the endothermic charging process leading to the breaking of bonds between the adsorbent and adsorbate. The reverse occurs during discharging process as the stored heat is evolved with the bonding of the adsorbate and adsorbent. Adsorption can also be classified as physisorption or chemisorption based on the nature 
of bonding involved. Physisorption involves electrostatic forces whereas chemisorption involves the formation of chemical bonds (Khalfaoui, Knani, Hachicha, \& Lamine, 2003). The heat can be stored for long periods of time as long as the components are separated (Dicaire \& Tezel). As the equilibrium of adsorption is dependent on temperature, adsorbents are able to adsorb more at lower temperatures (Chan, Chao, Sze-To, et al., 2012). Also, the type of adsorbent and adsorbate, the surface area of the adsorbent and conditions of the experiment affect the adsorption rate (White, 2012).

Thermodynamically, energy storage employing adsorption phenomena can be described as a heat pump process. As heat is stored and released based on the adsorption/desorption cycle, heat can be upgraded from low temperature heat to high temperature heat (Henninger, Jeremias, Kummer, Schossig, \& Henning, 2012). During the adsorption process, the heat stored is released as the adsorbate vapour bonds with the adsorbent surface. The adsorbate vapour returns to its liquid phase as it releases heat a certain temperature. However, the evaporation and adsorption of the adsorbate continues over the adsorption phase with the continuous release of heat. Thus, upgrading the heat from a low to high temperature level. It is important the TES system is evacuated before the process of storage. Competition from other gases present in the system are likely to affect the storage capacity of the adsorbent (Chan, Chao, \& Bahrami, 2012). During regeneration/desorption, high temperature heat is applied to remove the adsorbate. This cycle can be represented by the Clausius-Clapeyron Cycle as in Figure 3.

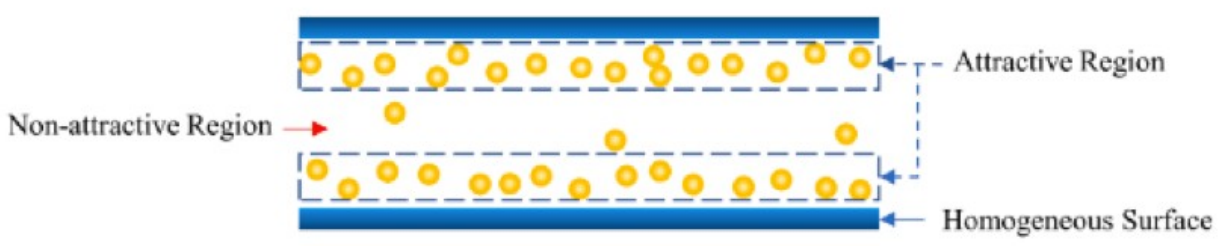

(a) Pore with homogeneous wall

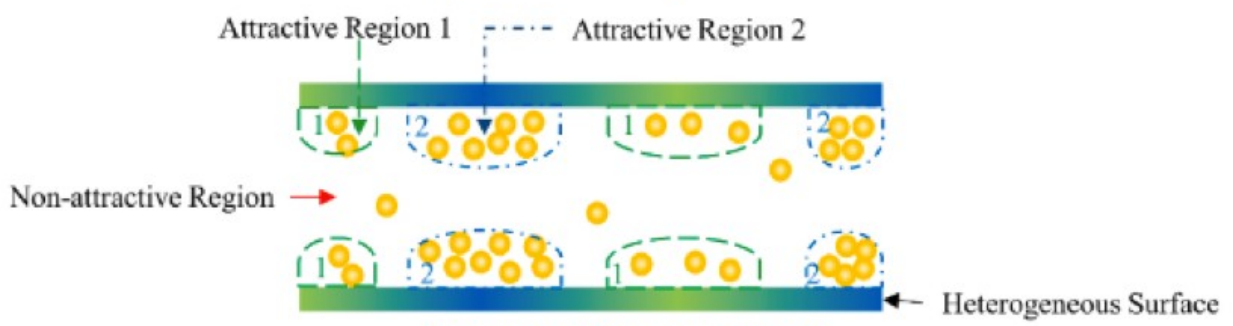

(b) Pore with heterogeneous wall

Figure 2. Attractive regions in pores of different surface properties with different adsorbate distributions (Kong \& Adidharma, 2019)

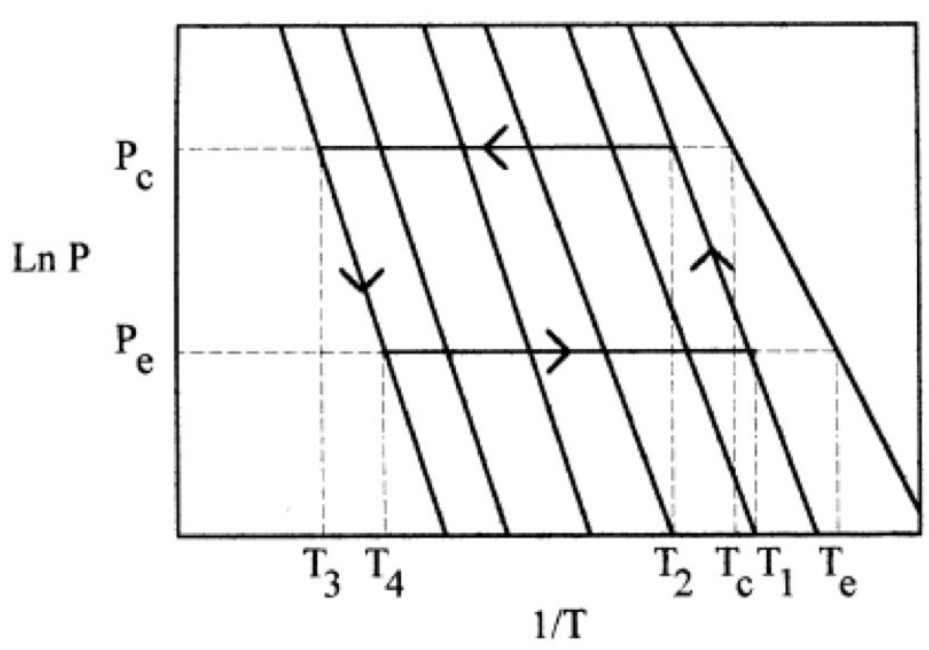

Figure 3 . The adsorption heat pump cycle (Thommes et al., 2015) 
The isosteric heat of adsorption which gives the amount of heat released is given by the Clausius-Clapeyron equation (Tchernev, 2001):

$$
d \operatorname{In} P / d T=H(T) / R T 2
$$

Where $\mathrm{P}$ is the gas pressure, $\mathrm{R}$ is the gas constant, and $\mathrm{T}$ is the zeolite temperature.

Zeolites are aluminosilicates with channels and pores that can be occupied by water molecules as well as other cations. A critical property is its reversible water adsorption properties (Adriano, Soriano, \& Duque, 2013). They have a stable three-dimensional structure and are able to adsorb molecules based on their sizes, shapes or electrical charges (Ityona, 2012). This makes them very useful in industrial applications. Their strong hydrophilic nature and ability to alter this property makes them better suited for TES applications than other adsorbent candidates such as silica gels. The adsorbate is bonded to the surface of the zeolite as in Figure 4.

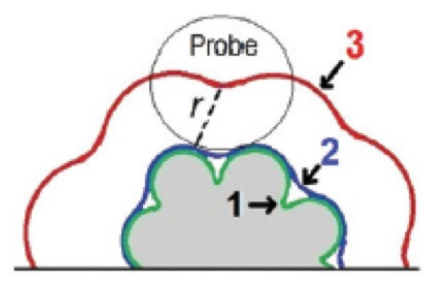

Figure 4. Schematic representation of several possible surfaces of an adsorbent. 1: van der Waals; 2: Connolly, Probe- accessible; 3: Accessible, r-distance (Thommes et al., 2015)

Zeolite A with $\mathrm{Si} / \mathrm{Al}$ ratio of 1 has about $47 \%$ open space is one of the simplest zeolites to synthesize (Ityona, 2012). Although the adsorption capacity of zeolites has been shown to vary with the presence of other molecules, they are good candidates due to their high thermal stability. They also store heat rather in chemical bonds between the adsorbent and adsorbate thus no significant energy losses (van Alebeek et al., 2018). They also have crystal lattices of regular sizes when compared to other adsorbents (Menad, Feddag, \& Rubeins, 2016).

The synthesis of zeolites from chemical reagents is expensive. The synthesis from natural or chemical reagents have similar chemical properties, pore size, thermal stability, higher purity and uniform particle size (Mgbemere, Ekpe, \& Lawal). The use of natural starting materials such as kaolin is ideal for the synthesis of Zeolite A (Gougazeh \& Buhl, 2014). The use of kaolin was started from the 1970s as it is transformed by calcination into a more reactive phase, metakaolin. The efficiency of a TES system is dependent on the amount of heat energy released during the adsorption stage (Dicaire \& Tezel). It is also important that the process is simple with the adsorption and desorption cycle. This process is applicable in storing solar thermal energy, geothermal energy and waste heat from industrial, commercial and domestic processes (Gopal, Hollebone, Langford, \& Shigeishi, 1982).

\section{Material and methods}

Zeolite A was synthesized using kaolin from Wassa, Ghana as starting material. The hydrothermal synthesis route used was a modification of (Von-Kiti, 2016). $1.75 \mathrm{~kg}$ of metakaolin (calcined at $600{ }^{\circ} \mathrm{C}$ ) following the procedure in Figure5.

Fusion of the metakaolin with the $\mathrm{NaOH}$ was done for 45 minutes at $600 o \mathrm{C}$. The crystallization of the slurry was done at $105^{\circ} \mathrm{C}$ for 24 hours. It is important that washing is done properly and the final product is not over-washed.

For TES, the objective is to capture the thermal energy for storage and release for use at a later period. The TES system designed and developed comprises an evaporator, an adsorber and a condenser connected by copper tubing and valves (Figure 6). 


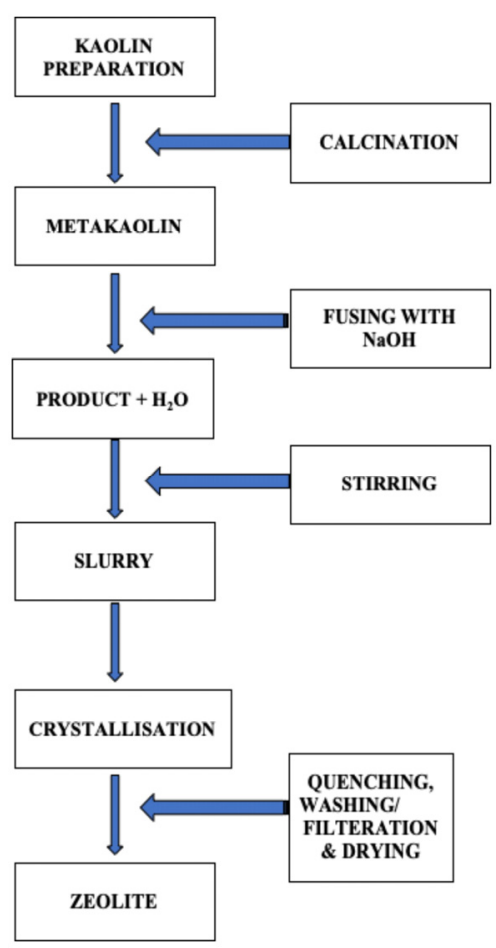

Figure 5. Synthesis process

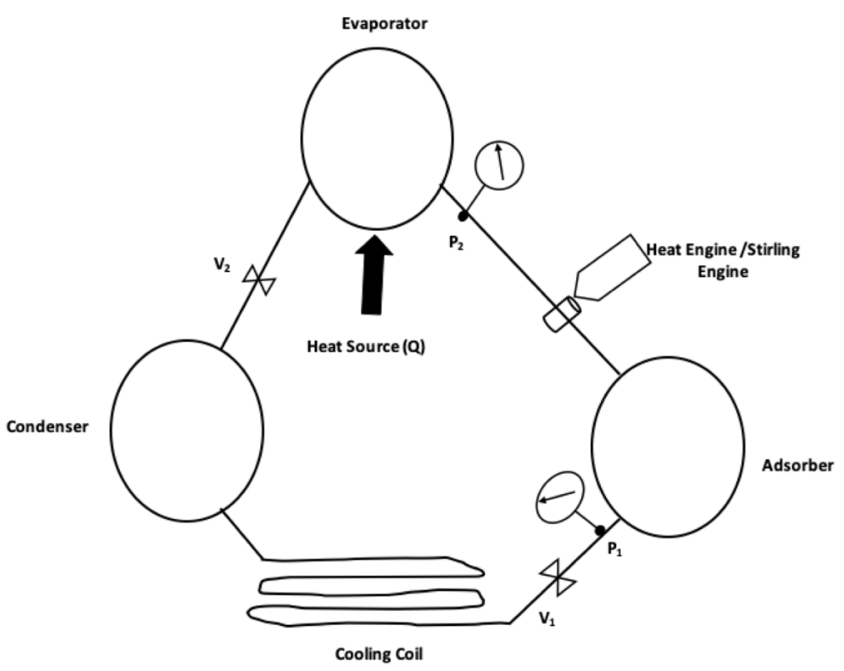

Figure 6. Schematic of the TES system

The zeolite was charged by heating the system to temperatures in the range of 95 to $105{ }^{\circ} \mathrm{C}$, the system was vacuumed. The opening of valve 1 began the desorption process with valve 2 closed. Water in the evaporator was heated using an LPG burner as external heat source. Temperature and pressure within the system was recorded as well as the mass of the zeolite. The reverse cycle of adsorption was achieved by opening valve 2 and closing valve 1. Water vapour was moved via capillary action from the condenser through the evaporator to the adsorber as zeolite is hygroscopic. The amount of water was measured to determine the amount adsorbed by the zeolite. The experimental parameters used are as in Table 2. 
Table 1: Experimental parameters

\begin{tabular}{lllll}
\hline Experiment & $\begin{array}{l}\text { Adsorbent } \\
\text { Amount }(\mathrm{g})\end{array}$ & Adsorbate Amount (g) & $\begin{array}{l}\text { Time } \\
(\text { mins) }\end{array}$ & Repetition (no) \\
\hline 1 & 100 & 150 & 60 & 5 \\
2 & 200 & 300 & 60 & 5 \\
3 & 300 & 450 & 60 & 5 \\
4 & 400 & 600 & 60 & 5 \\
5 & 500 & 750 & 60 & 5 \\
\hline
\end{tabular}

Studies of the properties of adsorbent materials have mostly been done via thermogravimetry, differential thermogravimetry, microcalorimetry as well as gravimetric isotherm measurements. The measured parameters of water vapour pressure $(\mathrm{P})$, temperature $(\mathrm{T})$, and water adsorption capacity $(\mathrm{n})$ was used to define the isosteric equilibrium curves as well as Langmuir isotherms for the zeolite - water system.

\section{Results and discussions}

Understanding the zeolites requires a couple of characterization techniques as a single method will not provide enough information. Scanning electron microscopy (SEM), X-ray diffraction (XRD) and Fourier-Transformed Infrared Spectroscopy (FTIR) were the techniques employed for the characterization of the zeolite synthesized.

Results obtained from characterization confirmed the synthesis of Zeolite A. The Zeolite A synthesized from the kaolin sourced from Wassa was of good quality as those synthesized from reagents. A comparison with the dehydrated zeolites indicated now significant structural changes as the peaks were at the same positions.

The adsorption process consists of two phases - the desorption and adsorption phases. The zeolite crystal structure, geometry and presence of acceptor centers contribute to the adsorption volume (Akimkhan, 2012). The maximum adsorbed amount of adsorbate at a specific pressure and temperature is defined as the equilibrium uptake (Rahman, Muttakin, Pal, Shafiullah, \& Saha, 2019). This can be represented with an isotherm model to show the varied uptakes with respect to pressure. The IUPAC has classified the various isotherms under physisorption as in Figure 10.

Adsorption-desorption isosteres were obtained from the temperature-pressure measurements. The adsorption isosteres were observed not to correlate as compared with the desorption experiments. The disparity may be attributed to the reasons as ascribed in (Herzog et al., 2014) with respect to the gas phase behaviour, sorption molar volume and desorbed amount. Based on the theory of volume micropore filling (TVMF), this disparity confirms the different amounts of adsorbate adsorbed by the zeolite. The use of adsorption isosteres makes it possible to study the process with only the physicochemical properties of the adsorbate as well as the structural and energetic constants of the adsorbent (Shkolin, Fomkin, \& Yakovlev, 2007). The linearity of the isosteres gives a more accurate calculation of the adsorption isotherms.

The angular coefficient of the calculated isosteres (trendline) exceeds that of the experimental as the calculated heat of adsorption exceeds the real heats of adsorption. However, they are similar in the desorption experiments. During adsorption, the micropores are being filled unlike in desorption. The trendlines are a representation the isosteres at equilibrium as they must satisfy the Clausius Clayperon equations (Tchernev, 2001). The slopes as observed were all greater than 1 indicating that the adoption process occurred as the adsorbed gas phase was in a lower energy state than the gas vapour.

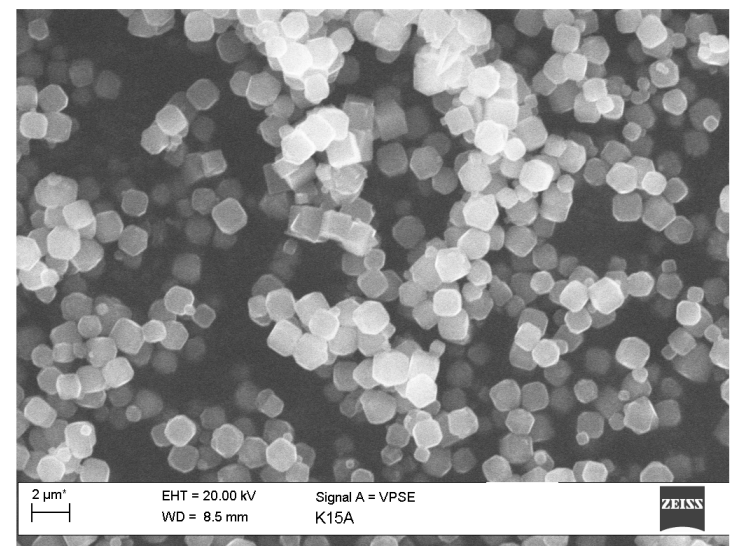

Figure 7. SEM image of Zeolite A 


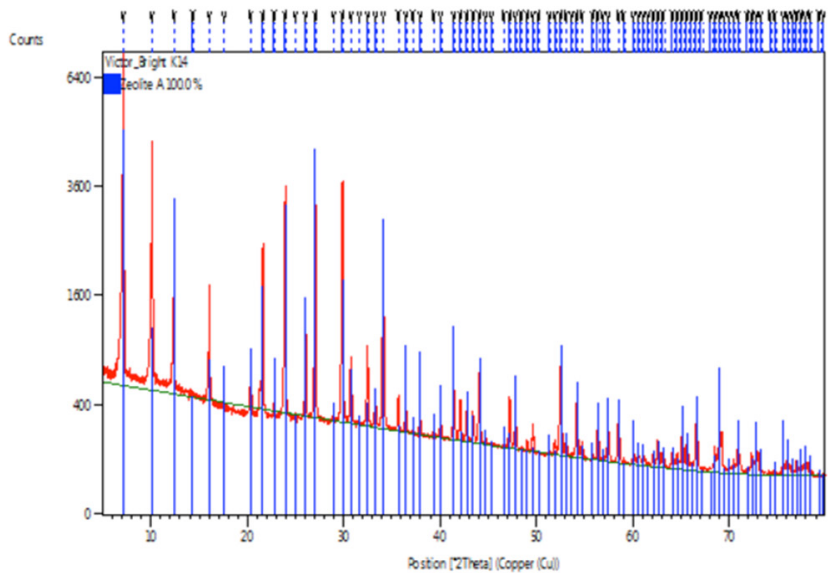

(a) Synthesized Zeolite A

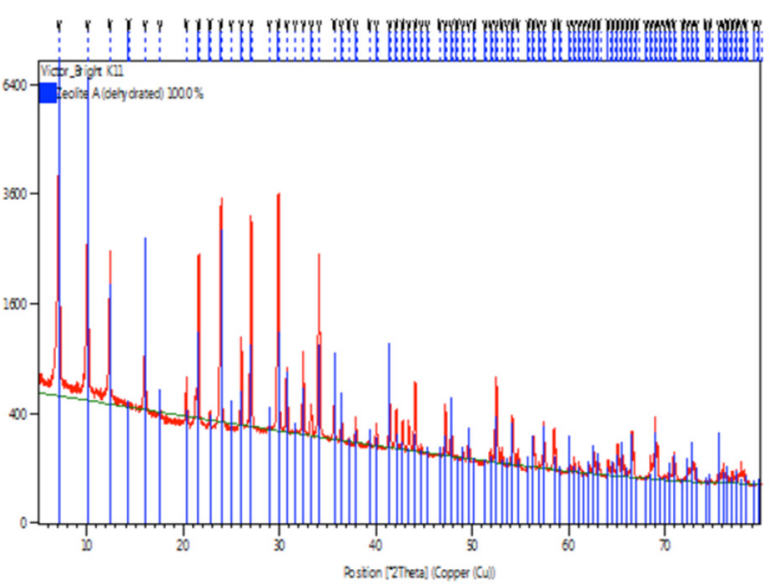

(b) Dehydrated Zeolite A

Figure 8. XRD image of Zeolite A

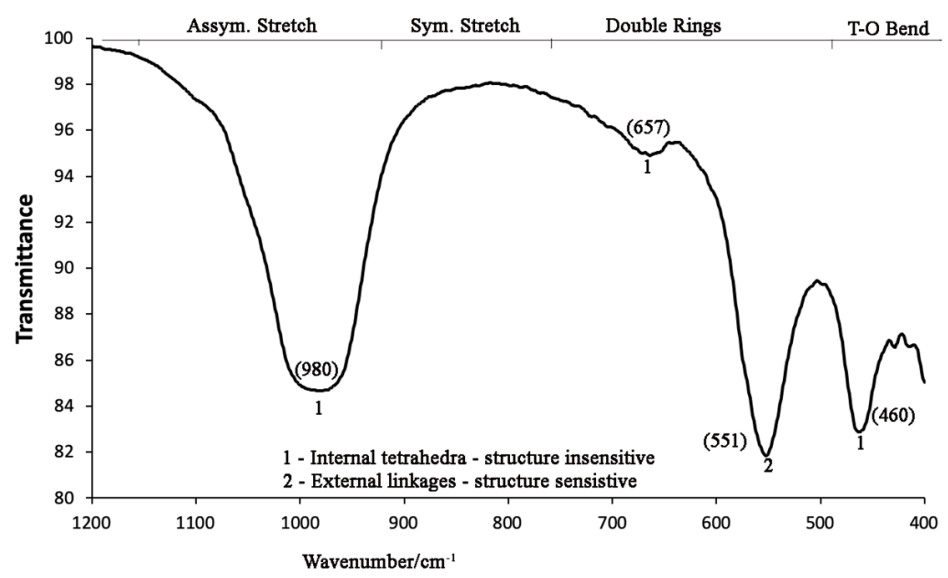

Figure 9. FTIR of Zeolite A

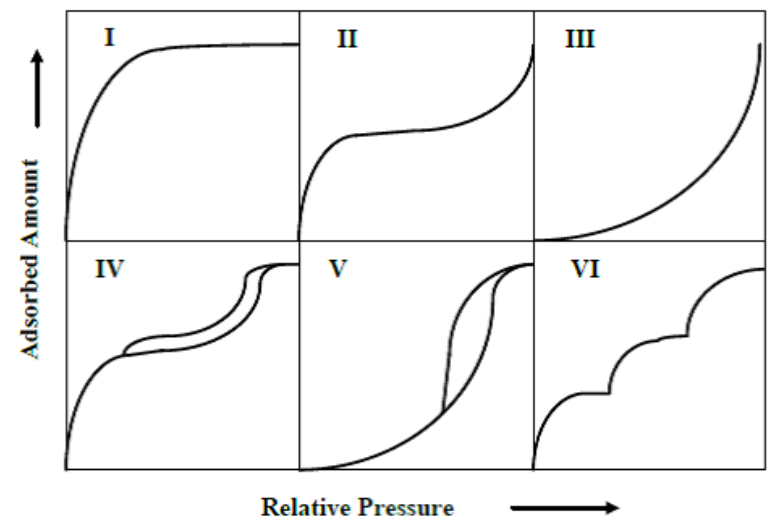

Figure 10. Classification of physisorption isotherms (Thommes et al., 2015) 

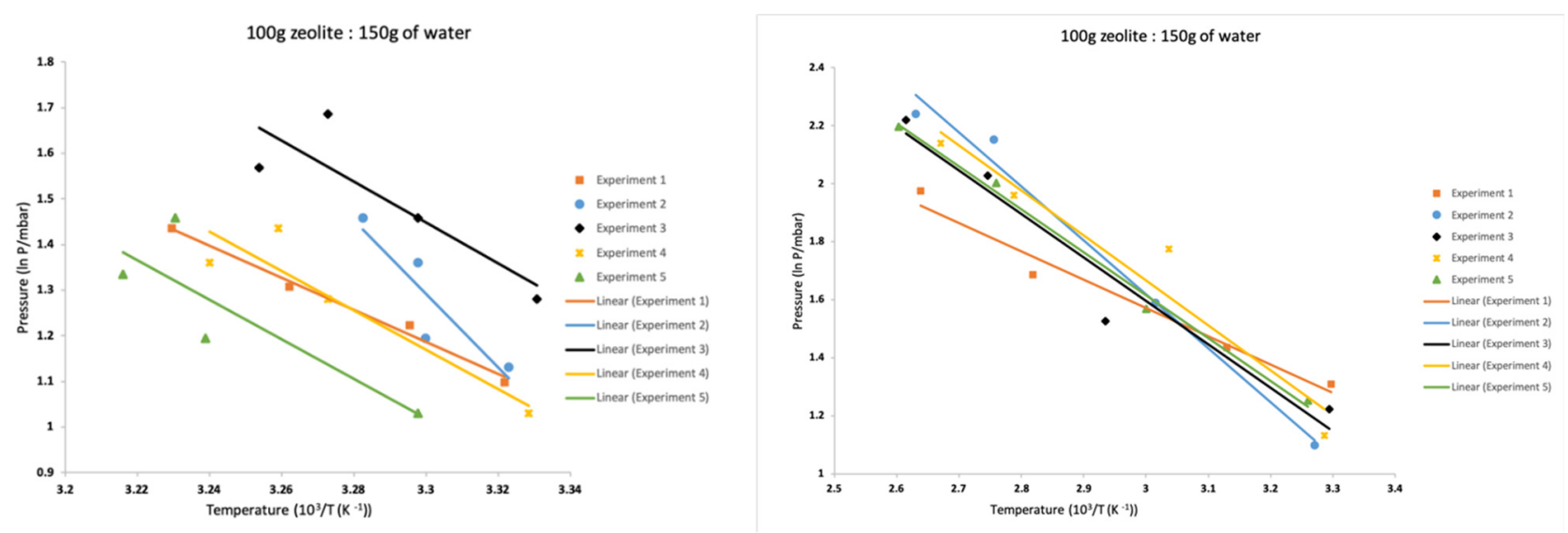

Figure 11. 100g Isosteres
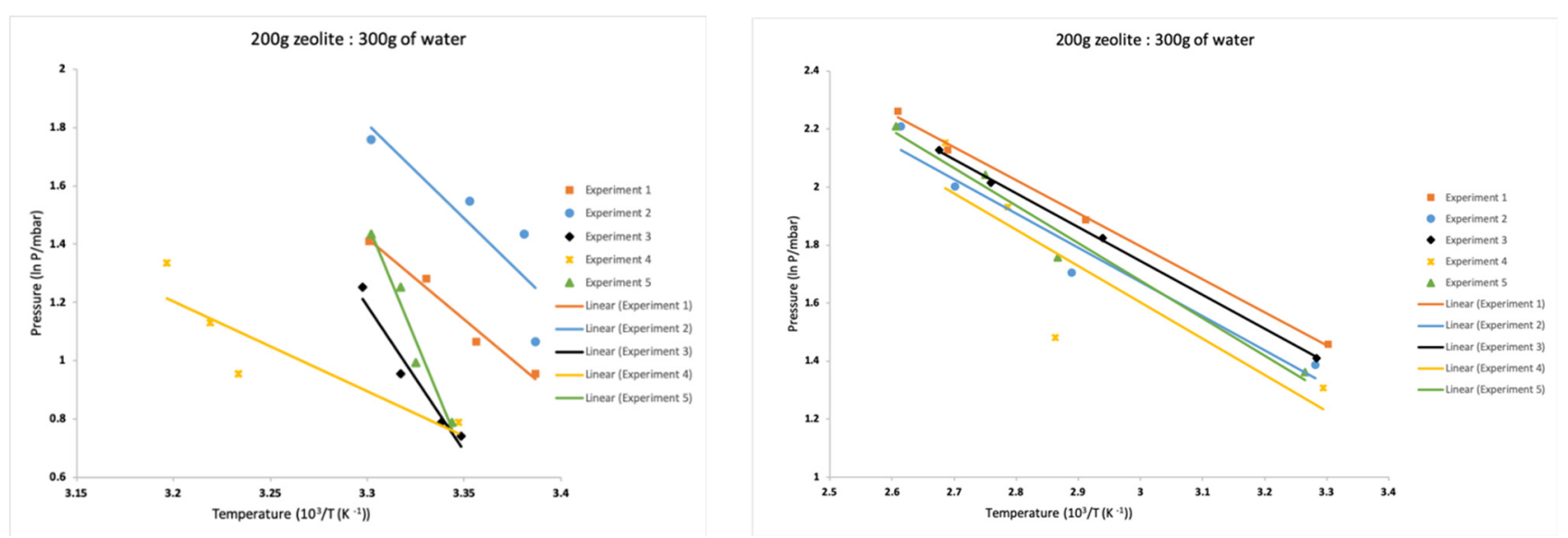

Figure 12. 200g Isosteres
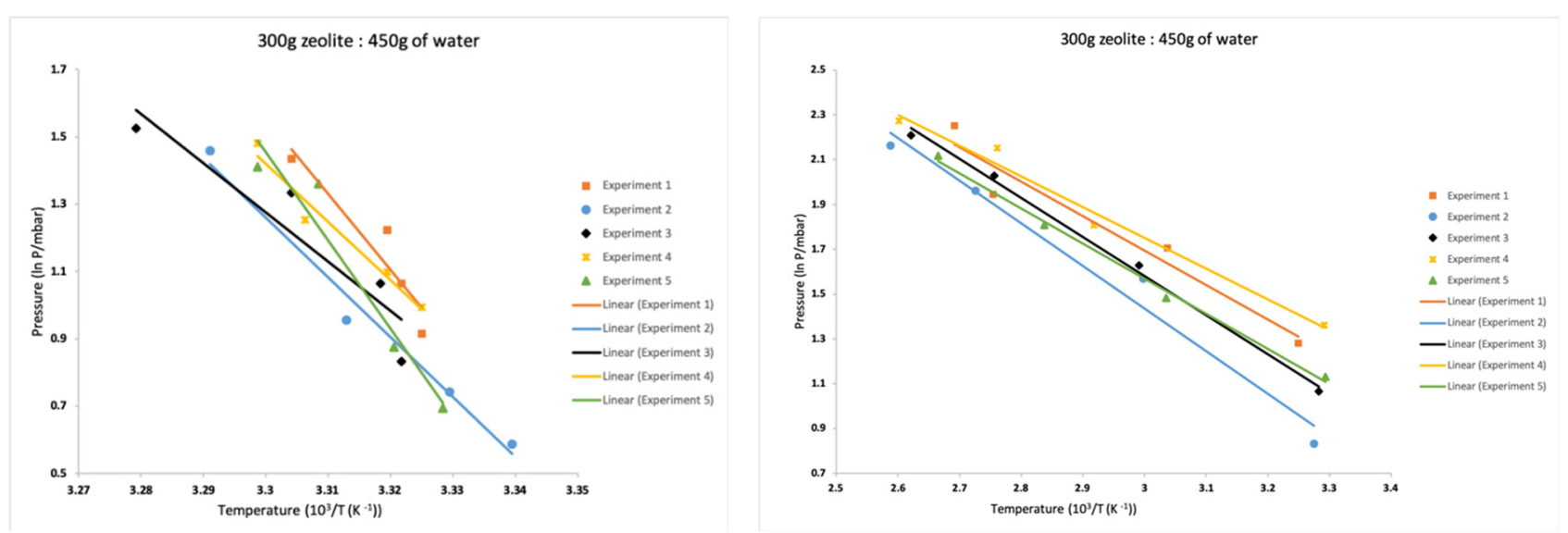

Figure 13. 300g Isosteres 

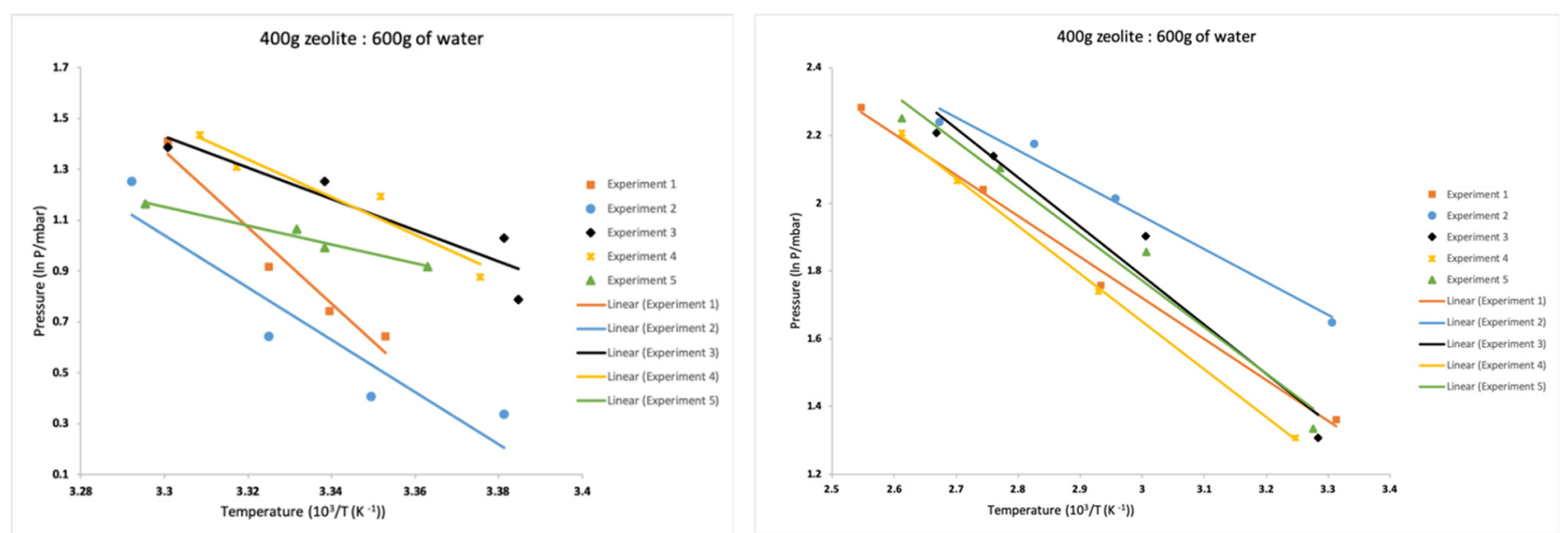

Figure 14. 400g Isosteres
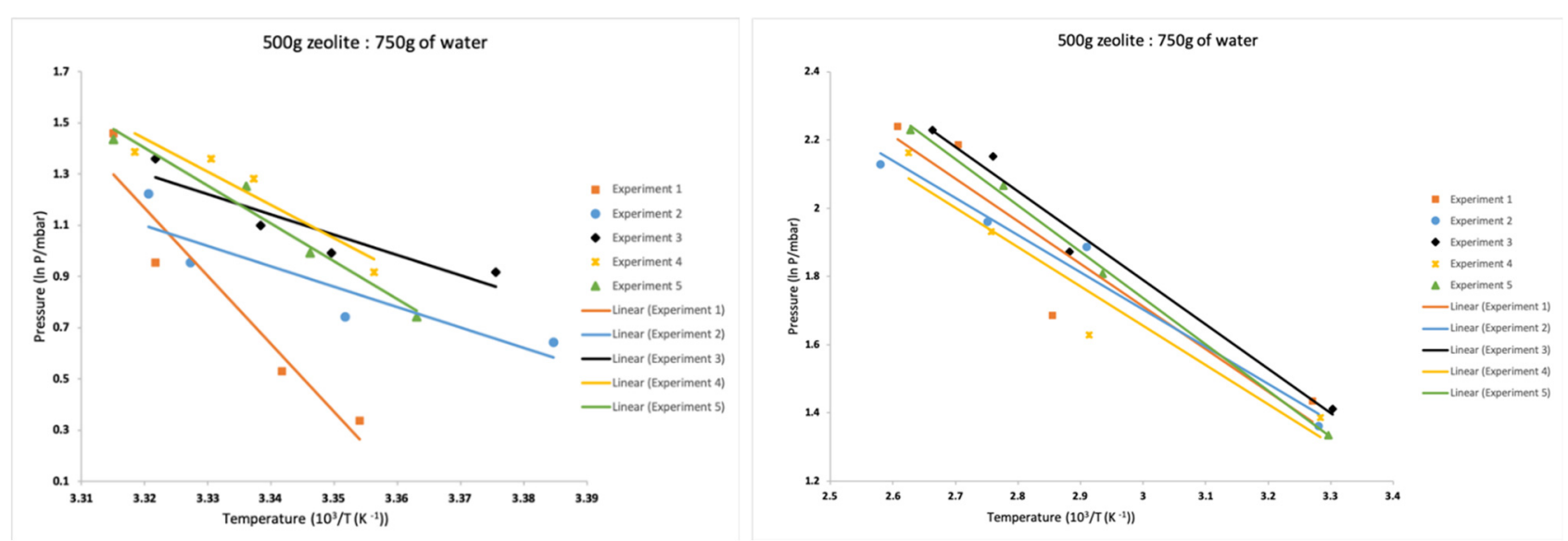

Figure 15. 500g Isosteres

The adsorption capacity is dependent on the interaction of the adsorbent and adsorbate as the adsorption characteristics is dictated by this interaction. Therefore, adsorbents with higher adsorption capacities tend to have higher selectivity, surface area and pore volume (Kulprathipanja, 2010). Zeolites with less Al and charge compensating cations per unit cell as in (J. Janchen \& Stach, 2011) tend to have less adsorption capacities.

At adsorption equilibrium, adsorption isotherms give information on the effect of heats of adsorption on the adsorbent surface (Chakraborty \& Sun, 2014). Although adsorption isotherms of zeolites adsorbing water are usually nonlinear as in Figure 10, that is Type 1, some forms of hysteresis have been observed during condensation (Ityona, 2012).

The Langmuir equation has been used in describing monolayer adsorption (Kong \& Adidharma, 2019; Kulprathipanja, 2010). Apart from the $400 \mathrm{~g}$ isotherm, R- squared values obtained were greater than $90 \%$. Although no best-fit model exists for Zeolite A - water pair adsorption, it is evident that the adsorption was favourable. In the application of the Langmuir model, it is assumed that the adsorbent is homogenous and that each adsorption site is occupied by only one atom (Rahman et al., 2019). 

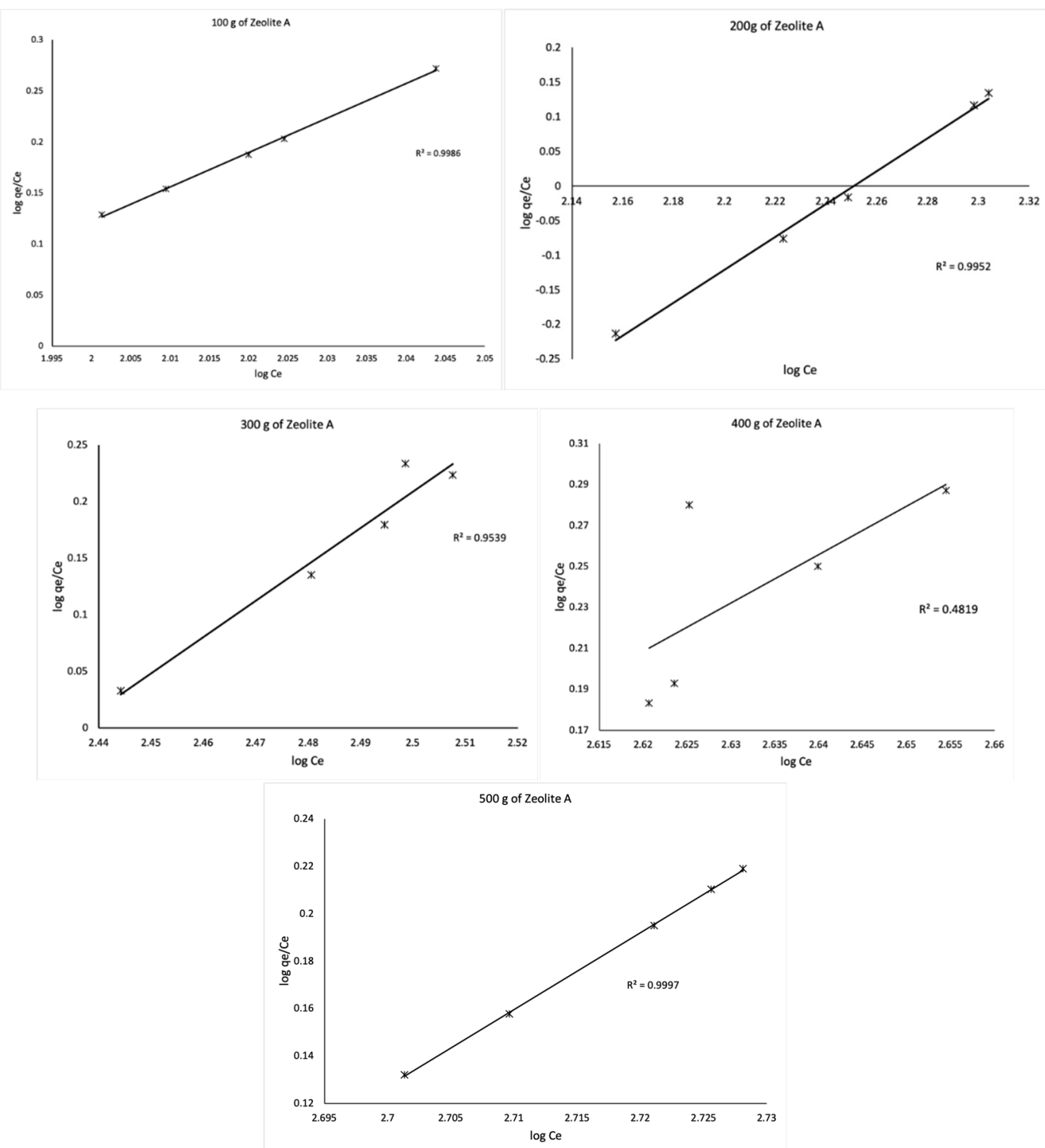

Figure 16. Langmuir Isotherms

Thermodynamic analysis of the synthesized zeolite A from kaolin is momentous. The derivation of the adsorption isosteres contribute to the rapid analysis of the adsorption process. This is through the relationship of the isosteric heat of adsorption at any temperature and pressure and the latent heat vaporization of the adsorbate to the slope of the isostere (Tchernev, 2001). Thus, the isosteric heat of adsorption is equal to the latent heat of vaporization multiplied by the slope as in Figure 17. 2,400 J/g was used as heat of vaporization of water found in the steam tables in (Tchernev, 2001).

In comparison with the maximum adsorption capacity (qm) as in Table 2, there is an inverse relationship. $100 \mathrm{~g}$, $300 \mathrm{~g}$ and $500 \mathrm{~g}$ zeolites which had lower maximum adsorption capacities have higher isosteric heats of adsorption than $200 \mathrm{~g}$ and $400 \mathrm{~g}$ zeolites obvious from their calculations. However, the adsorption capacity is not enough to explain the discrepancies observed. These may be caused by the lack of attainment of equilibrium or changes in samples during sample dehydration (Vieillard, 2010). 


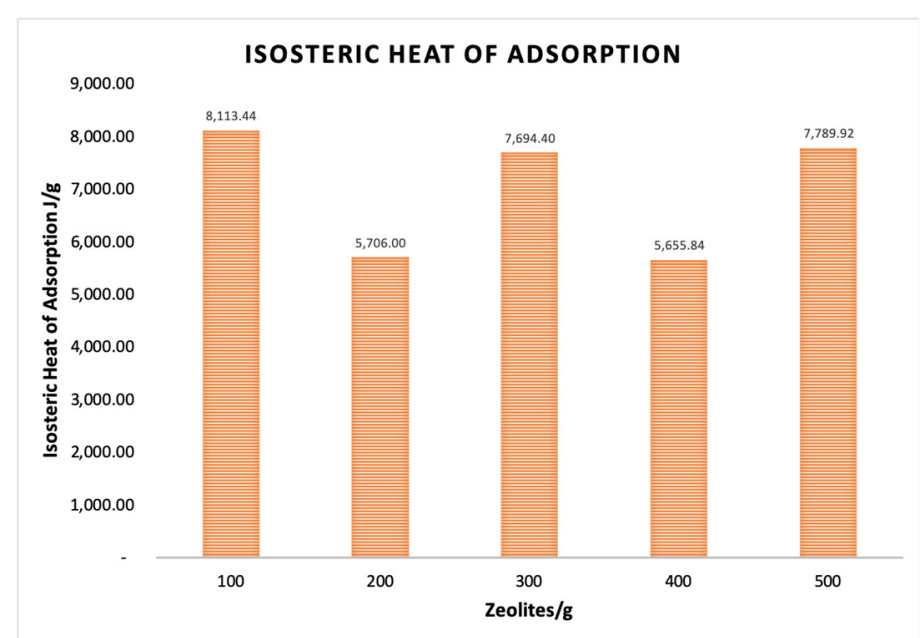

Figure 17. Comparison of the isosteric heats of adsorption

Table 2. Langmuir isotherm constants

\begin{tabular}{lllll}
\hline Zeolite & $\mathrm{qm}(1 / \mathrm{slope}) / \mathrm{g} / \mathrm{g}-1$ & $\mathrm{~b}(1 / \mathrm{y}$-intercept $) / \mathrm{g}-1$ & slope & y-intercept \\
\hline 100 & 0.295805478 & 0.509210863 & 3.3806 & 6.6389 \\
200 & 0.420609884 & 0.444267962 & 2.3775 & 5.3515 \\
300 & 0.311915159 & 0.410657103 & 3.206 & 7.807 \\
400 & 0.424340151 & 0.395024892 & 2.3566 & 5.9657 \\
500 & 0.308090455 & 0.375814837 & 3.2458 & 8.6367 \\
\hline
\end{tabular}

The energy of adsorption, $b$, decreases with increasing zeolite amount. This indicates the decreasing affinity for the zeolite by the water vapour. This could be caused by the increasing competition for adsorption sites or nonattainment of equilibrium conditions. The increasing amount of zeolite also limits the diffusion of the adsorbate within the system. The tendency of zeolites to agglomerate at the surface contributes greatly to this. Thus, increasing zeolite thickness leads to a slower adsorption kinetics (Dawoud, 2013) and lead to longer cycle times for the attainment of equilibrium (Chan, Chao, \& Bahrami, 2012). To reduce the effect of agglomeration at the surface, the use of the lowest possible grain size would be effective. This, however, may require an infinite number of heat exchanger fins (Dawoud, 2013). Consequently, leading to the increase the thermal mass of the adsorber and require more energy to heat the system.

To improve the performance of a TES system, the adsorption system should have better heat and mass transfer $(\mathrm{Li}$, 2013). Adsorbate uptake during the adsorption and desorption phases at equilibrium is analogous to the system performance (Chan, Chao, Sze-To, et al., 2012). Eliminating heat storage losses is also imperative in improving the system. Some forms of modifications to improve on the adsorbent bed includes the use of granules and composite adsorbents (White, 2012). Increasing charging temperature also contributes to increasing energy storage density (Ding \& Riffat, 2013). This, nonetheless, also leads to heat losses in the system. For a continuous system, heat losses are minimal unlike systems that require long term storage of heat for which only the heat of adsorption will be available.

\section{Conclusion}

Utilizing zeolites can contribute to solving some significant problems of the technology and the environment. Zeolites synthesized from kaolin have been used in other applications. The thermodynamic analysis of some aspects of zeolite synthesized from Wassa kaolin gives more information on its use in TES applications. The microstructure as obtained using characterization techniques such as SEM, FTIR and XRD confirmed the synthesis of Zeolite A.

Isosteres and Langmuir isotherms for zeolites of amounts 100, 200, 300, 400 and $500 \mathrm{~g}$ were obtained from temperature and pressure readings. This was similar to isosteres of known zeolites. This enabled the analysis of the thermal dependence of the zeolite adsorbent and water vapour adsorbate.

The results obtained showed that the zeolite synthesized from kaolin could be used in TES applications. The isosteric heat of adsorption obtained ranged from 5,655.84 to 8,113.44 J/g.

Thus, it is possible to reduce the cost of zeolites whiles increasing the application value of zeolites. 


\section{References}

Adriano, A., Soriano, G., \& Duque, J. (2013). Characterization of water absorption and desorption properties of natural zeolites in Ecuador. Paper presented at the Fifth International Symposium on Energy, Puerto Rico Energy Center-Laccei.

Akimkhan, A. M. (2012). Structural and ion-exchange properties of natural zeolite. Ion Exchange Technologies, 261-282.

Asamoah, R. B., Nyankson, E., Annan, E., Agyei-Tuffour, B., Efavi, J. K., Kan-Dapaah, K., . . Yaya, A. (2018). Industrial applications of clay materials from Ghana (a review). Oriental Journal of Chemistry, 34(4), 17191734. doi: $10.13005 / \mathrm{ojc} / 340403$

Chakraborty, A., \& Sun, B. (2014). An adsorption isotherm equation for multi-types adsorption with thermodynamic correctness. Applied Thermal Engineering, 72(2), 190-199. doi:10.1016/j.applthermaleng.2014.04.024

Chan, K. C., Chao, C. Y. H., \& Bahrami, M. (2012). Heat and mass transfer characteristics of a zeolite $13 X / \mathrm{CaCl2}$ composite adsorbent in adsorption cooling systems. The Hong Kong University of Science and Technology, Hong Kong, China Simon Fraser University, Surrey, BC, Canada M4 - Citavi. https://asmedigitalcollection.asme.org/ES/proceedings/ES2012/44816/49/232042

Chan, K. C., Chao, C. Y. H., Sze-To, G. N., \& Hui, K. S. (2012). Performance predictions for a new zeolite $13 \mathrm{X} / \mathrm{CaCl} 2$ composite adsorbent for adsorption cooling systems. International Journal of Heat and Mass Transfer, 55(11-12), 3214-3224. doi:10.1016/j.ijheatmasstransfer.2012.02.054

Dawoud, B. (2013). Water vapor adsorption kinetics on small and full scale zeolite coated adsorbers; A comparison. Applied Thermal Engineering, 50(2), 1645-1651. doi:10.1016/j.applthermaleng.2011.07.013

Dicaire, D., \& Tezel, F. H. (2009). Study of Adsorbent Energy Density and Regeneration for Long Term Thermal Energy Storage Of Solar and Waste Heat: University of Ottawa.

Ding, Y., \& Riffat, S. B. (2013). Thermochemical energy storage technologies for building applications: a stateof-the-art review. International Journal of Low-Carbon Technologies, 8(2), 106-116. doi:10.1093/ijlct/cts004

Gopal, R., Hollebone, B. R., Langford, C. H., \& Shigeishi, R. A. (1982). The rates of solar energy storage and retrieval in a zeolite-water system. Solar Energy, 28(5), 421-424. doi:10.1016/0038-092X(82)90261-4

Gougazeh, M., \& Buhl, J. C. (2014). Synthesis and characterization of zeolite A by hydrothermal transformation of natural Jordanian kaolin. Journal of the Association of Arab Universities for Basic and Applied Sciences, 15(1), 35-42. doi:10.1016/j.jaubas.2013.03.007

Gwadera, M., \& Kupiec, K. (2014). A Silica Gel-water Adsorption System with an Adsorbent-coated Copper Pipe.

Henninger, S. K., Jeremias, F., Kummer, H., Schossig, P., \& Henning, H. M. (2012). Novel sorption materials for solar heating and cooling. Energy Procedia, 30, 279-288. doi:10.1016/j.egypro.2012.11.033

Herzog, T. H., Jänchen, J., Kontogeorgopoulos, E. M., \& Lutz, W. (2014). Steamed zeolites for heat pump applications and solar driven thermal adsorption storage. Energy Procedia, 48, 380-383. doi:10.1016/j.egypro.2014.02.044

Ityona, A. (2012). Experimental determination of the adsorption capacity of synthetic Zeolite A/water pair for solar cooling applications. Journal of Mechanical Engineering Research, 4(4). doi:10.5897/jmer11.080

Janchen, J., Schumann, K., Thrun, E., Brandt, A., Unger, B., \& Hellwig, U. (2012). Preparation, hydrothermal stability and thermal adsorption storage properties of binderless zeolite beads. International Journal of LowCarbon Technologies, 1-5.

Janchen, J., \& Stach, H. (2011). Adsorption properties of porous materials for solar thermal energy storage and heat pump applications. Energy Procedia.

Khalfaoui, M., Knani, S., Hachicha, M. A., \& Lamine, A. B. (2003). New theoretical expressions for the five adsorption type isotherms classified by BET based on statistical physics treatment. Journal of Colloid and Interface Science, 263(2), 350-356. doi:10.1016/S0021-9797(03)00139-5

Khan, G., Arafat, S., Reza, M., Razzaque, S., \& Alam, M. (2010). Linde Type-A zeolite synthesis and effect of crystallization on its surface acidity. Indian Journal of Chemical Technology, 17, 303- 308. 
Kong, L., \& Adidharma, H. (2019). A new adsorption model based on generalized van der Waals partition function for the description of all types of adsorption isotherms. Chemical Engineering Journal, 375, 122112-122112. doi:10.1016/j.cej.2019.122112

Kulprathipanja, S. (2010). Zeolites in Industrial Separation and Catalysis. KGaA, Weinheim: WILEY-VCH Verlag GmbH Co.

Li, G. (2013). Review of thermal energy storage technologies and experimental investigation of adsorption thermal energy storage for residential application. (Master's thesis). University of Maryland Libraries,

Menad, K., Feddag, A., \& Rubeins, K. (2016). Synthesis and study of calcination temperature influence on the change of structural properties of the LTA zeolite. RASAYAN. J. Chem, 9(4), 788-798.

Mgbemere, H. E., Ekpe, I. C., \& Lawal, G. I. (2017). Zeolite synthesis, characterization and application areas: A review. International Research Journal of Environmental Sciences, 6(10), 45-59.

Paksoy, H. O. (2005). Thermal Energy Storage for Sustainable Energy Consumption. Amsterdam: Springer.

Rahman, M. M., Muttakin, M., Pal, A., Shafiullah, A. Z., \& Saha, B. B. (2019). A statistical approach to determine optimal models for IUPAC-classified adsorption isotherms. Energies, 12(23), 4565-4565. doi:10.3390/en12234565

Scapino, L., Zondag, H. A., Van Bael, J., Diriken, J., \& Rindt, C. C. M. (2017). Energy density and storage capacity cost comparison of conceptual solid and liquid sorption seasonal heat storage systems for low-temperature space heating. Renewable and Sustainable Energy Reviews, 76, 1314-1331. doi:10.1016/j.rser.2017.03.101

Schreiber, H., Lanzerath, F., Reinert, C., Grüntgens, C., \& Bardow, A. (2016). Heat lost or stored: Experimental analysis of adsorption thermal energy storage. Applied Thermal Engineering, 106, 981-991. doi:10.1016/j.applthermaleng.2016.06.058

Shkolin, A. V., Fomkin, A. A., \& Yakovlev, V. Y. (2007). Analysis of adsorption isosteres of gas and vapor on microporous adsorbents. Russian Chemical Bulletin, 56(3), 393-396. doi:10.1007/s11172-007-0064-6

Tchernev, D. I. (2001). Natural zeolites in solar energy heating, cooling, and energy storage. Reviews in Mineralogy and Geochemistry, 45(1), 588-617. doi:10.2138/rmg.2001.45.17

Thommes, M., Kaneko, K., Neimark, A. V., Olivier, J. P., Rodriguez-Reinoso, F., Rouquerol, J., \& Sing, K. S. W. (2015). Physisorption of gases, with special reference to the evaluation of surface area and pore size distribution (IUPAC Technical Report). Pure and Applied Chemistry, 87(9-10), 1051-1069. doi:10.1515/pac2014-1117

van Alebeek, R., Scapino, L., Beving, M. A. J. M., Gaeini, M., Rindt, C. C. M., \& Zondag, H. A. (2018). Investigation of a household-scale open sorption energy storage system based on the zeolite $13 \mathrm{X} /$ water reacting pair. Applied Thermal Engineering, 139, 325-333. doi:10.1016/j.applthermaleng.2018.04.092

Vieillard, P. (2010). A predictive model for the entropies and heat capacities of zeolites. European Journal of Mineralogy, 22(6), 823-836.

Von-Kiti, E. (2016). Synthesis and characterization of zeolites from bauxite and kaolin: application to the removal of heavy metals from mining wastewater. (Doctoral dissertation). Kwame Nkrumah University of Science and Technology,

White, J. (2012). A CFD simulation on how the different sizes of silica gel will affect the adsorption performance of silica gel. Modelling and Simulation in Engineering, 2012, 1-12. doi:10.1155/2012/651434

\section{Copyrights}

Copyright for this article is retained by the author(s), with first publication rights granted to the journal.

This is an open-access article distributed under the terms and conditions of the Creative Commons Attribution license (http://creativecommons.org/licenses/by/4.0/). 